This item was submitted to Loughborough's Research Repository by the author.

Items in Figshare are protected by copyright, with all rights reserved, unless otherwise indicated.

\title{
Working towards a holistic organisational systems model
}

PLEASE CITE THE PUBLISHED VERSION

http://dx.doi.org/10.1109/SYSOSE.2010.5544113

PUBLISHER

(c) IEEE

VERSION

VoR (Version of Record)

LICENCE

CC BY-NC-ND 4.0

REPOSITORY RECORD

Hubbard, Ella-Mae, Carys E. Siemieniuch, Murray A. Sinclair, and Allan Hodgson. 2019. "Working Towards a Holistic Organisational Systems Model". figshare. https://hdl.handle.net/2134/7138. 
This item was submitted to Loughborough's Institutional Repository (https://dspace.lboro.ac.uk/) by the author and is made available under the following Creative Commons Licence conditions.

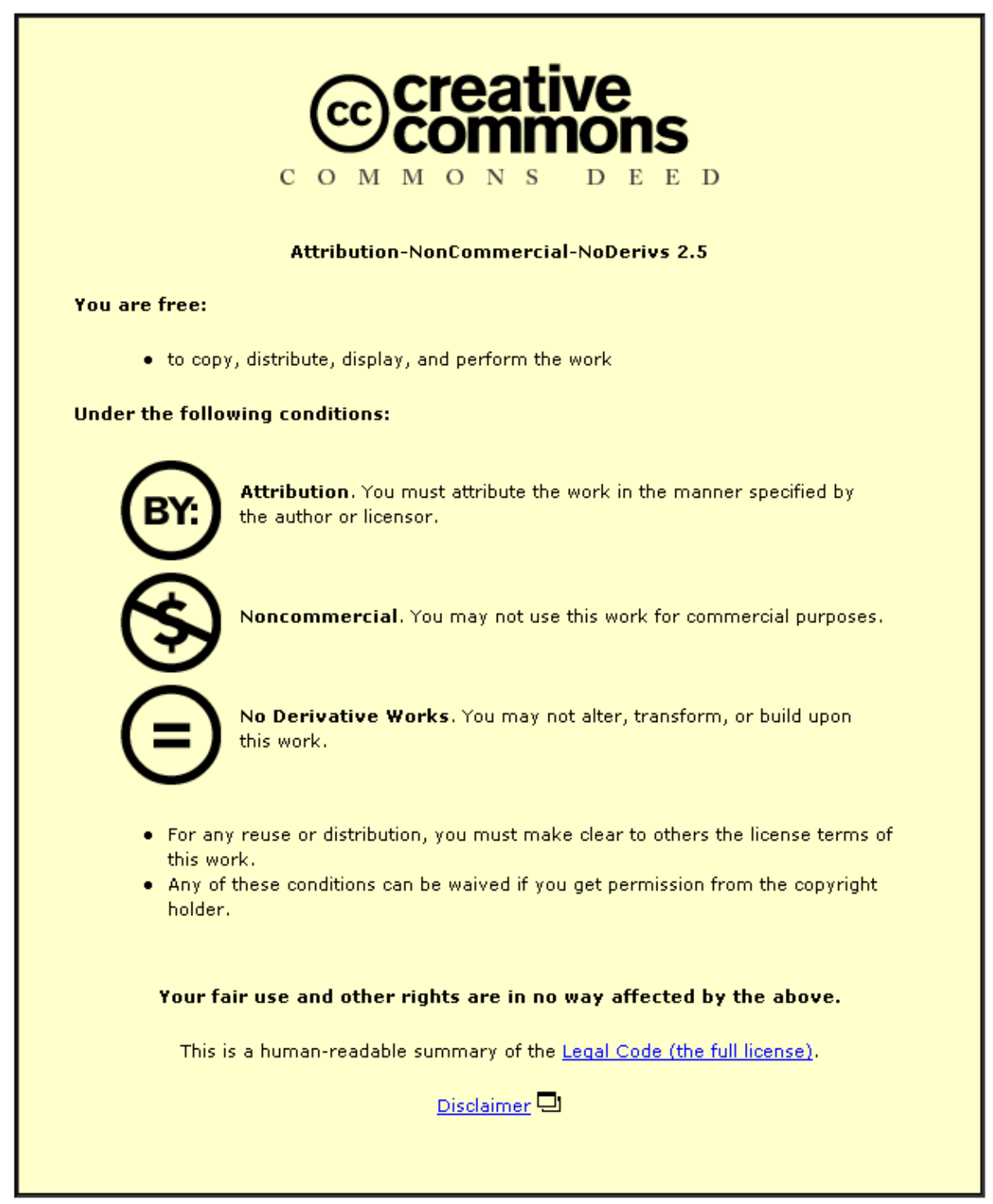

For the full text of this licence, please go to: http://creativecommons.org/licenses/by-nc-nd/2.5/ 


\title{
Working towards a holistic organisational systems model
}

\author{
E-M Hubbard ${ }^{1}$, C. E. Siemieniuch ${ }^{1}$, M. A. Sinclair ${ }^{2}$, A Hodgson $^{1}$ \\ ${ }^{1}$ Department of Electronic and Electrical Engineering, \\ ${ }^{2}$ Centre for Innovative and Collaborative Engineering, Department of Civil and Building Engineering \\ Loughborough University, Leicestershire, LE11 3TU, UK
}

\begin{abstract}
This paper presents an integration effort combining a number of soft factors modelling tools and considers the potential impact of such an overall tool in a system of systems environment. The paper introduces the tools developed and how it is envisaged they will work together to provide a comprehensive, coherent output. It is suggested that a suite of interoperable tools of this form could aid the design and operation of organisational systems and ensure they are fit for purpose.
\end{abstract}

Enterprise Systems Modelling, Organisational Systems Engineering, Simulation, Organisational Performance

\section{INTRODUCTION}

$\mathrm{T}$ he primary focus of the authors' current work is in the area of Enterprise System Modelling and more particularly in the domain of 'soft' or human/organisational aspects of these System Models. Enterprise modelling (EM) is concerned with the representation and specification of the various aspects of enterprise operations, namely: functional aspects to describe what are the things to be done and in which order; informational aspects to describe which objects are used or processed; resource aspects to describe what or who performs things and according to which policy; and organisational aspects to describe the organisational structure and the responsibility frame within which things are being done [1].

A useful summary of Enterprise Architectures is found in $[2,3]$, but existing models and reference architectures (e.g. (GERAM [4], VERAM [5], ToVE [6], PERA [7]) tend to deal with enterprise elements such as Resources, Information Flows and Functions well, but a) within a process framework and b) they do not show a sufficient capability to include 'soft' enterprise characteristics such as policies, culture, competencies, decision making structures etc. within dynamic models. Hence, changes in one or more of these characteristics are not shown in overall organisational system performance.

It is generally accepted that organisational systems (large/ small, temporary/ permanent etc) need better integration strategies and (re)configurable organisational architectures if they are to be able to achieve faster response times, improved decision making processes, flexibility/adaptability in the face of change, improved resilience, etc.. In addition, there is a need for tools and methods to overcome the barriers [8] that affect our ability to understand and model complex systems and their emergent behaviours. The toolset presented in this paper goes some way towards addressing these issues. The authors term their approach to developing this capability as Organisational Systems Engineering.

\section{ORGANISATIONAL SYSTEMS ENGINEERING}

Organisational Systems Engineering (OSE) involves treating the enterprise as a system, made up of human, process and technical sub-systems, which interact and interface with each other. As argued previously, there is a need to be able to model all elements of the system, (including in this case 'softer' organisational characteristics such as role interactions, cultural values, knowledge distribution, competencies, decision-making systems, enterprise strategy and team reliability), in order to aid the understanding of the impact of internal and external change on interactions within and between enterprise sub-systems.

\section{A. Why do we want to model enterprises?}

There remains a severe lack of usable, integrated dynamic enterprise simulation models that allow organisations to explore prior to deployment the implications of change initiatives such as the introduction of new processes, new capabilities, new working practices etc, particularly from an organisational and human performance perspective. The holy grail of being able to look into the future by evaluating the effectiveness, impact or added value of alternative organisational system configurations, prior to deployment, is still a long way off. Such a capability would greatly enhance an organisation's ability dynamically to (re) configure appropriate systems (people, process and technology) to achieve the performance required to produce designated output in different contexts and to avoid structures that are susceptible to adverse circumstances such as accidents, disasters and undesirable emergent behaviour.

Enterprise models allow a way to visualize, represent and analyse the inner workings of an organisation or enterprise. Where a change or transition is being experienced, enterprise modelling can provide insight into problems, diagnose symptoms, identify and compare alternatives and develop a plan for the future. In general, an enterprise model provides a common basis for discussion, allowing an opportunity to improve performance and increase profit - given the current economic climate around the world, any competitive edge could be very valuable for the organisations involved.

\section{B. System of Systems (SoS)}

As noted in a previous paper [9], a SoS environment enhances some of the challenges for organisations and necessarily for organisational systems engineering and enterprise modelling: issues arising include the need for organisational agility, the impact of induced and intrinsic complexity, elimination of undesirable emergent behaviour, 
and more efficient knowledge and information management systems. The multi-disciplinary approach taken by the authors is also founded on an SoS point of view and hence takes into account the interlinking of issues, which arise with through life capability considerations. In addition since global collaboration requirements require extensive supply constellations to work in partnership, any work in this area must also focus on better alignment of organisational strategies, processes and structures throughout the supply chain.

\section{OVERVIEW OF TOOLSET}

This paper introduces work carried out by the Engineering Systems of Systems (ESoS) Research Group at Loughborough University [10]: the aim of this group is to research the interoperability, sustainability and reconfigurability of socio- technical Systems of Systems (SoS) to improve their predictability and usability. To this end the group had to develop an emerging portfolio of tools. that together enable an organisation to assess how it is organised to achieve its goals. The purpose of this exercise is to create a 'bigger picture' representation of an organisational system. Such an organisational systems model may help provide input to questions such as 'are we doing the right things' and 'are we doing those things right', providing critical input to 'Engineering Governance'.

The current toolset is represented in Figure 1 below and comprises 5 main tools/methods.

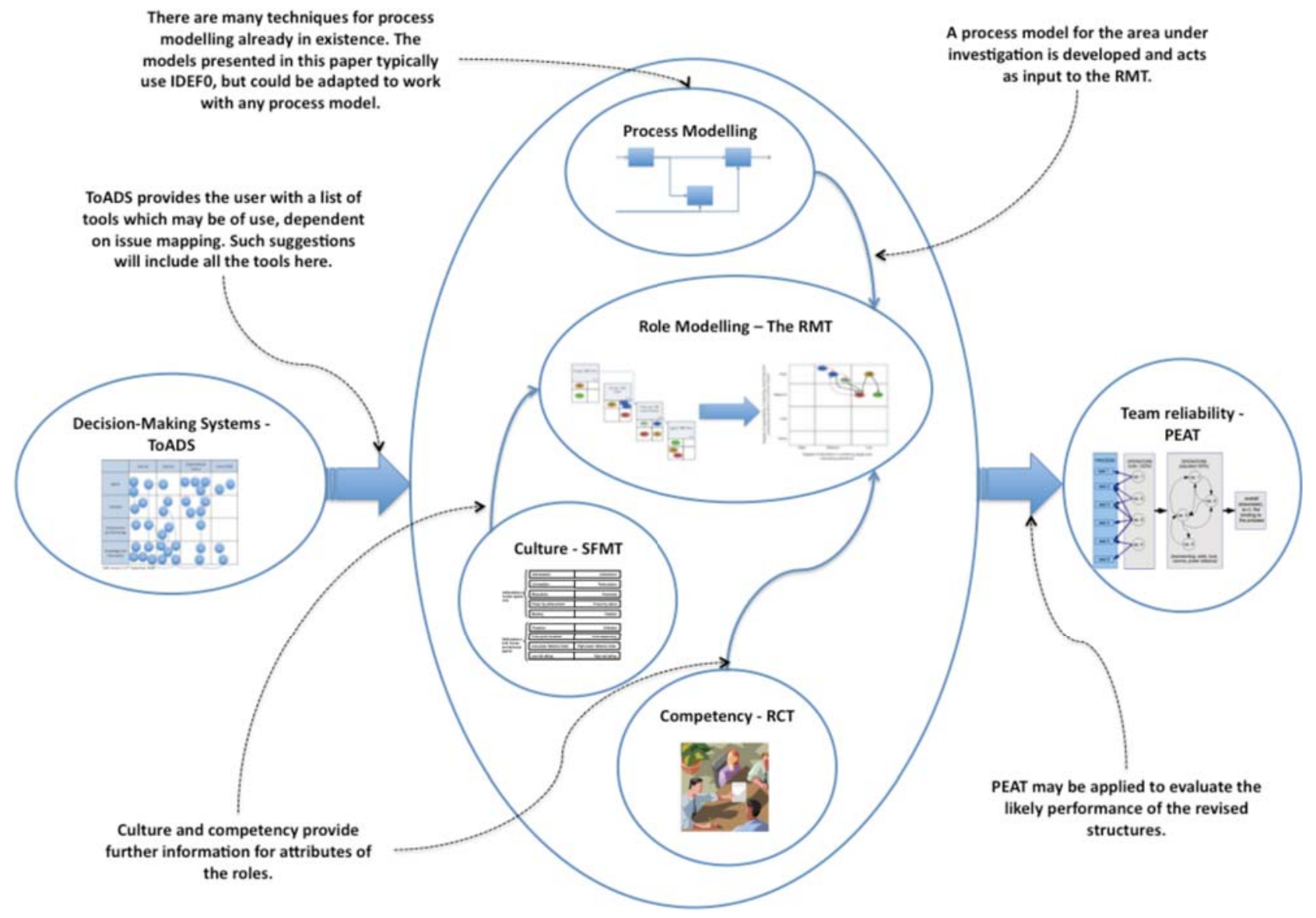

Fig 1 Overview of ESoS toolset

- $\quad$ RMT (Role Matrix Technique): a relatively easy and quick method which enables process owners, project managers and other practitioners to analyse, evaluate and select the most appropriate combination of human roles (class, profile, boundaries, interactions, authority and responsibilities) for a given process(es). It can also be used to explore organisational structures, providing insight into the sources of the emergent role behaviour that frequently impairs organisational performance
- SFMT (Soft Factors Modelling Tool): allows users to evaluate whether a particular configuration of assets (technical and human) are capable of demonstrating appropriate decision-making, information processing, communication, adaptive skills and behaviour in an environment where, for example, the command style is control free, authority is delegated, operational tempo is unpredictable and the context is ill defined. 
- KCT (Knowledge Configuration Tool): put simply the $\mathrm{KCT}$ will explore the knowledge requirements for a staffing plan emerging from the RMT.

- $\quad$ ToADS (Tool for the Assessment of Decision-making Systems): assists organisations in evaluating and (re)configuring their DMS (Decision-Making Systems), to help them cope with the risks and opportunities of long life, complex, engineered projects and systems - using the tool stakeholders are able to analyse and investigate DMS in their organisations to establish where and why they may not be working efficiently.

- PEAT (Performance Evaluation and Assessment for Teams): this tool is capable of predicting the likelihood of success of a team executing a system process and is likely to be used by systems engineers in the initial stages of systems design when concepts are still fluid.

One key constraint underpinning the development of all these tools is that they should be simple to use, negating the need for consultants. Due to space constraints only three tools from the portfolio are described in this paper: the Role Matrix Technique (RMT), the Soft Factors Modelling Tool (SFMT) and the Tool to Assess Decision-making Systems (Toads): PEAT is described in more detail in [11]. However the authors hope to run a tutorial on the whole portfolio during the conference

\section{Role MATRiX TECHNIQUE}

The research group have been developing a technique called the Role Matrix Technique (RMT) for over a decade [12]. In the beginning the RMT was intended to provide a relatively easy and quick method, which enables process owners, project managers and other practitioners to analyse, evaluate and select the most appropriate combination of human roles (class, profile, boundaries, interactions, authority and responsibilities) for a given process. In essence, given a process outlined as a flow diagram (e.g. from an IDEF0 analysis [13]) the RMT will enable a staffing plan to be created and enable a visual analysis of possible organisational problems that will be encountered if that particular staffing pan is executed. Over the years, it has expanded to become a means to explore and illuminate organisational structures, showing how roles fit together (or not), and providing some insight into the sources of the emergent behaviour that reduces so much of organisational performance.

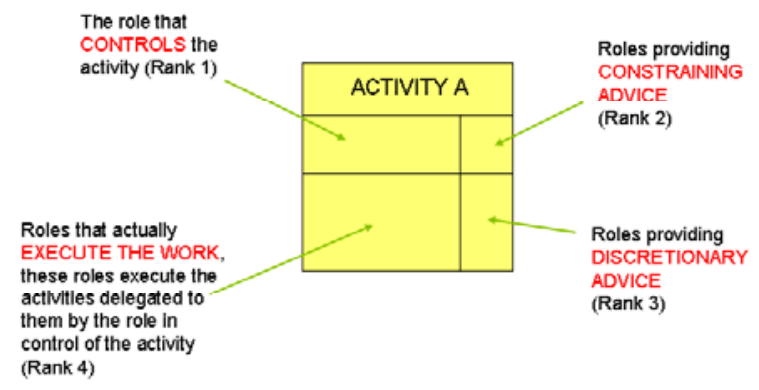

Fig 2 The RMT quadrants

The RMT has two key elements: the first enables the representation of the nature of involvement of different kinds of roles for a given activity, as shown in Fig. 2 .

Roles are allocated to an activity within an overall process, depending on whether their contribution is in controlling or executing the activity or providing constraining or discretionary advice. There is only ever one main role in control of any activity, but there may be any number of roles executing or providing advice.

Constraining advice is typically that which comes down the organisational hierarchy, but not from those in the direct line of responsibility. Discretionary advice typically is that which arises from Communities of Practice, which may extend

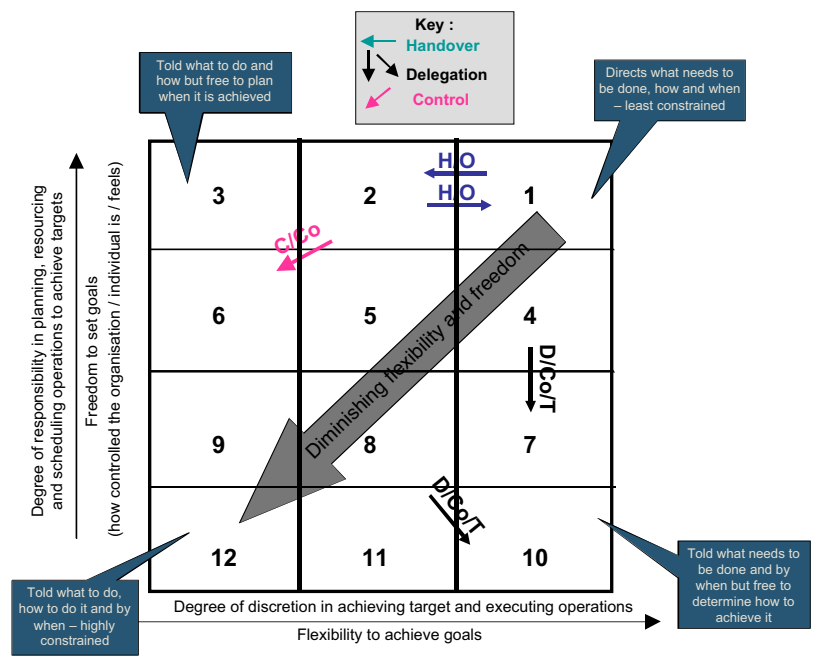

beyond the organisation.

Figure 3: RMT Matrix

The second element of the RMT is the Role Matrix, an overview of which is provide in Figure 3. The horizontal axis of the Role Matrix represents how much discretion a role holder has in carrying out the actual operations / tasks which have been allocated to them; and the vertical axis is intended to represent how much discretion a role holder has in terms of planning, resource allocation and scheduling the operation of other role holders, or in deciding what, how and when operations allocated to them are carried out.

When using the Role Matrix and positioning roles within the different grid sections, the analyst is essentially 'describing' or 'positioning' each role relative to the dimensions of the gird and relative to other roles also placed in the grid. There are rules for translating from the RMT Quadrants to the RMT Matrix which are linked to their position in the RMT quadrants for each of the activities in the process(es) being modelled. There are also identified relationships between each of the cells in the RMT Matrix, as indicated in the Key in Figure 3 above and by postioning 2 roles in different cells the key relationships between them are identified and captured.

As a technique the RMT is fairly resource intensive: software support to generate the graphics, capture and analyse assumptions made etc would vastly improve the tool's ease of 
use and some prototypes are under development. Versions of the RMT have been applied in a range of companies from military contexts to smaller process and manufacturing organisations.

\section{TOADS}

The aim of Toads (Tool for the Assessment of Decisionmaking Systems) is to assist organisations in configuring their DMS (Decision-Making Systems), to help them cope with the risks and opportunities of long life, complex, engineered projects and systems. Toads is described in more detail in [14] but a brief summary is provided here.

The four constituent parts of a DMS (an extension of the classic people, process and technology view) are :

- Agents: Decision Making (DM) agents may be human or software based and are involved in the DM process.

- Activities: DM activities are those activities necessary for a decision to be made, creating the DM process.

- Infrastructure and technology: DM infrastructure and technology is that which enables and provides support for the DM process.

- Knowledge and information: That which is necessary for a decision to be made.

DM knowledge and information flows through the infrastructure and technology, around the DM activities to the agents to allow decisions to be made. There are also four further variables that may impact or be impacted by the DMS:

- Internal variables: contextual issues, e.g. what stage are you at in the lifecycle and what impact does this have.

- Environmental variables: external influences e.g. legislation and health and safety or the interface with external partners or the supply chain.

- Organisational culture: issues such as power distance (structure and empowerment), risk (how much risk are the agents and the organisation willing to take), regimentation (what is mandated/standard practice) and collaboration (individual work vs. collaborative work).

- Level of DM: Strategic, tactical or operational.

Together, the four parts of the DMS and four impacting variables form the rows and columns respectively of a key component of Toads: the Decision Making Framework (DMF). Users can locate problems that emerge during an investigation on the DMF and then diagnose deeper issues. A snapshot view of the DMF is given at Figure 4 showing the four DMS elements as the row headings and the first of the four impacting variables as a column heading.

The tool has been evaluated in several case studies in different domains (engineering, construction, manufacturing) and interesting trends have emerged: decisions are not always consciously made: people cannot actively look for support for something they are unaware they are doing and nor will they communicate these decisions. There is also a tendency to adapt (sub-optimally) existing processes for new projects rather than develop new ones.

With regard to the onward development of Toads the authors recognize that there are some issues that the tool does not currently address. These include training and workload,

\begin{tabular}{|c|c|}
\hline Feature of DMS & Issue \\
\hline \multirow{3}{*}{ A. Agents/ Roles } & Poor role/ agent definition \\
\hline & Poor role/ agent allocation \\
\hline & Non-availability of roles/ agents \\
\hline \multirow{2}{*}{ B. Activities } & Inappropriate activities \\
\hline & $\begin{array}{l}\text { Poor definition of activities (unclear } \\
\text { or fuzzy boundaries) }\end{array}$ \\
\hline \multirow{2}{*}{$\begin{array}{l}\text { C. Infrastructure } \\
\text { and Technology }\end{array}$} & Inappropriate infrastructure \\
\hline & Non-availability of infrastructure \\
\hline \multirow{2}{*}{$\begin{array}{l}\text { D. Knowledge } \\
\text { and Information }\end{array}$} & $\begin{array}{c}\text { Inappropriate knowledge and/ or } \\
\text { information }\end{array}$ \\
\hline & $\begin{array}{l}\text { Non-availability of knowledge and/ or } \\
\text { information }\end{array}$ \\
\hline
\end{tabular}

Figure 4: Toads Decision Making Framework snapshot

albeit the framework can be used to investigate problems in these areas. Furthermore, the use of the Toads and associated processes will rely heavily on successful, honest identification of issues and subsequent categorisation. Given that few people are able to understand more than a small part of their own organisation, and given the power relationships and politics to be found in any organisation, these are difficult goals to achieve. However integration of Toads within the overall portfolio will help in providing a wider system view of the organisation.

\section{CUlture}

While the RMT and Toads provide structural diagnosis, this is not enough. It is also necessary to consider the organisation's culture. This is accomplished using the Soft Factors Modelling Tool (SFMT) [15], which evaluates organisational culture at three levels: organisation, group/team, and individual.

In its current form the SFMT is intended to support mission planners to evaluate a set of human and technical resources that they have brought (or propose to bring) together to carry out a mission in a particular environment. In order to evaluate the proposed resources, the user enters relevant information about the agents' cultures (at the organisational, team and individual levels) and the operational requirements of the mission or environment. To do this, the user scores each agent type in terms of nine cultural dimensions (as shown in figure 5) and defines the operational environment and the associated agent behavioural/skill requirements by selecting from a set of predefined options.

The SFMT then compares the agents' cultural factor scores 
against the ideal agent cultural factor scores for the defined operational environment and for the desired behavioural/skill requirements. It then calculates individual, average and overall average discrepancy scores; discrepancies are then highlighted using a 'traffic light' system - red for high discrepancy, amber/orange for moderate discrepancy and green for low discrepancy.
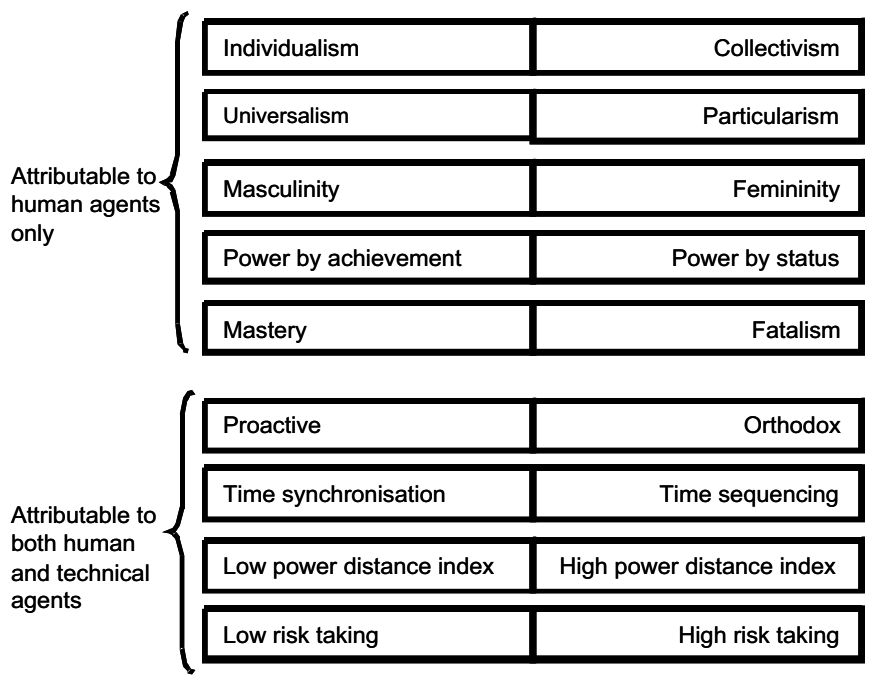

Figure 5: Nine cultural dimensions

The SFMT tool could be utilized in order to answer a question such as the following:

"Is the selected configuration of military assets capable of demonstrating appropriate decision-making, communication and adaptive skills and behaviour in an operational environment where the command style is control free, authority is delegated, operational tempo is unpredictable and the battlespace is ill-defined?"

The SMFT identifies mismatches between available resources and the demands of both the tasks that must be executed and the task environment:, thus adding depth to the diagnostics arising from the RMT and Toads. It is believed that as a result organisational change towards a better utilisation of the available human resources will become easier to plan and accomplish. The SFMT has been validated in both military and industrial case studies.

\section{OTHER AREAS}

Two other tools are under development. The first is a Knowledge Configuration Tool (KCT). This is premised on the notion that one can treat an organisation as a knowledge engine, which both captures knowledge to carry out its mission, and realises that knowledge in the capabilities that it delivers. Consequently, for efficiency, it is necessary to ensure the configuration of knowledge (i.e. which role should know what, and what knowledge can be embedded in the process) across the organisation is at least appropriate.

The second is the Performance Evaluation and Assessment for Teams (PEAT) tool. This is a predictive tool; having used the other tools to diagnose the issues, and then having generated a possible to-be structure, PEAT can then be applied to evaluate the likely performance of the revised structures.

\section{INTEGRATING THE TOOLS}

Current work is focusing on the combination and integration of this suite of tools into a single, holistic tool, so that there would be a seamless transfer of work from one tool to another, in whatever order the user thinks fit. Given the work of the group, it has also been identified as a requirement that provision should be made to enable the integration of new tools or allow tools to be updated as research and development continues.

Figure 1 above shows how the various tools could work together. Each of the tools at the moment are stand alone and individually allow the capabilities of the individual resources that make up an enterprise or organisation to be assessed to varying degrees. It is much more difficult to assess, especially prior to deployment, the performance capability of the whole, integrated enterprise resource and particularly the human components, working collaboratively as a team. The challenge is to develop an integrated approach to define and evaluate [pre and post any planned change] whether the configuration(s) of human resources identified/selected is capable of making decisions and carrying out identified tasks in pursuit of an identified goal-set efficiently and effectively, given the constraints of a particular type of operational environment. Ideally this will be encapsulated in a decision support system which will include a simulation capability and would allow users to identify and evaluate alternative organisational configurations:

- RMT: a set of roles (definition, accountability, authority, responsibility and interactions) for a given process(es)

- SFMT: the cultural attributes and values required by the roles to deliver the required performance in a given operational or commercial context

- KCT: the knowledge requirements for the roles

- PEAT: prediction of the likelihood of success for Teams within the role structures

- Toads: evaluation of the Decision Making System within the role structures

This would allow managers to explore organisational system models with alternative configurations and improve project processes by having the ability to evaluate whether a particular organisational context could inhibit or facilitate the introduction of a new capability.

Furthermore, as mentioned earlier in this paper, global collaboration requirements require extensive supply constellations to work in partnership. The ability to model individual organisations within these supply constellations would enable two key benefits: to compare and contrast organisational strategies, processes and structures with the aim of indentifying conflicts that would impact on the ability of organisations to work seamlessly together; and secondly the ability to model the whole organisational structure of the supply chain at the strategic, tactical and operational level. This 'System of System' consideration will be even more important with the shift of responsibility, which comes with 
the growth in consideration of through life capability.

\section{CONCLUSION}

Before embarking on any large organisational systems modelling, it is important to remember that development and interpretation will be context dependent and will vary depending on the aim and objectives of the organisation or system of systems.

Models can be developed to explain simply what is going on with an organisation, to identify lessons learned or best practice or to tackle a specific problem such as issues regarding training, human resources or a specific process phase. A significant challenge, particularly for engineering organisations, is the shift to the provision of through-life capability support [16]. This will affect companies in their internal organisation and will demand changes to the processes employed. Such new processes will require different people and resources perhaps from a broader spectrum of national, organisational and professional cultures, including a wider pool of available and accessible knowledge. These processes must be accepted and integrated into existing/ legacy organisational systems if they are to be effective. An integrated organisational systems modelling and analysis tool could help facilitate such an integration. Considerations from agility suggest a paradigm shift is required, including elements notoriously difficult to change, such as culture and trust. Understanding how these things permeate through an organisation (and indeed beyond the an individual organisation throughout the supply chain) may help organisations avoid continual and non-value added series of organisational restructuring.

\section{ACKNOWLEDGMENT}

The research underpinning the toolset arose from four key projects, the first three funded by the EPSRC through the Innovative Manufacturing and Construction Research Centre (IMCRC) and the fourth through the Ministry of Defence funded UK Defence Technology Centre Systems Integration and Integrated Systems for Defence: Autonomous and SemiAutonomous Systems. In addition an industrially funded Engineering Doctorate focused on the development of a tool for human reliability assessment for teams.

\section{REFERENCES}

[1] F B Vernadat, "Enterprise modelling and integration - Principles and Applications" London, Chapman and Hall, 1996

[2] C E Siemieniuch and M A Sinclair, "Systems Integration - the 'System of Systems Issues". International Journal of Applied Ergonomics special issue on "Fundamental Reviews in Applied Ergonomics", eds J Wilson, R haslam \& p Carayon. Vol 37, pp91-110, 2006

[3] T J Williams, P Bernus, J Brosvic, D Chen,, G Doumeingts., L Nemes,, J L Nevins, B Vallespir., J Vlietstra, D Zoetekouw. "Architectures for Integrating Manufacturing Activities and Enterprises. Computers in Industry” 24 (2-3), Special Issue on CIM pp111-140, 1994

[4] IFIP-IFAC Task Force. (1999) The Generalised Enterprise Reference $\begin{array}{llll}\text { Architecture and } & \text { Methodology. } & \text { V } & 1.6 .3\end{array}$ http://www.cit.gu.edu.au/ bernus

[5] A Zwegers, M Tolle, J Besterager, "Virtual Enterprise Reference Architecture and Methodology (VERAM)". Joint D41/D43 deliverable of IST EU Project: Global Engineering and Manufacturing in Enterprise Networks (GLOBEMEN), Baan Development/Technical University of Denmark, 2002

[6] M S Fox, "The TOVE Project - Towards and Common Sense Model of the Enterprise". Report from the Enterprise Integration Lab, University of Toronto, 1992

[7] T J Williams, "The Purdue Enterprise Reference Architecture". Computers in Industry, 24(2), 141-158, 1994

[8] Advanced Technologies for Interoperability of Heterogeneous Enterprise Networks and their Application, EU Project no 507849. DELIVERABLE D.A1.1.1: First Version of State of the Art in Enterprise Modelling Techniques and Technologies to Support Enterprise Interoperability, Ana Belén García Díez , July, 2004

[9] E M Molloy, C E Siemieniuch and M A Sinclair, "Fit For Purpose Organisations in a System of Systems Environment", 7th Annual Conference on Systems Engineering Research, Loughborough, 2009.

[10] http://www.lboro.ac.uk/departments/el/research/systems/esos/index.html $\#$ tab=profile

[11] M A Sinclair, C E Siemieniuch, M J d C Henshaw, R A Haslam and L Evans L, "Predicting the performance of teams in systems". To be published in proceedings of $28^{\text {th }}$ Conference on Systems Engineering Research, March 17 -19, Hoboken, NJ, 2010

[12] K Callan, C E Siemieniuch and M A Sinclair, "A case study example of the role matrix technique", International Journal of Project Management, 24, pp. 506-515, 2006.

[13] Announcing the Standard for INTEGRATION DEFINITION FOR FUNCTION MODELING (IDEF0), Draft Federal Information, Processing Standards Publication 183, 1993 December 21

[14] A Hodgson, C E Siemieniuch and E-M Hubbard, "The Effects of Individual and Team Culture on Complex Systems.". To be published in proceedings of $28^{\text {th }}$ Conference on Systems Engineering Research, March 17 -19, Hoboken, NJ, 2010

[15] R Oliva and R Kallenberg, "Managing the transition from products to services", International Journal of Science Industry and Management, 14, pp. 160-172, 2003. 